\title{
Tetrahydrofuran Clathrate Hydrate Formation
}

\section{Conrad, Heiko}

2009

Conrad, H, Lehmkuhler, F , Sternemann, C, Sakko, A, Paschek , D , Simonelli , L , Huotari , S , Feroughi, O , Tolan, M \& Hämäläinen, K 2009 , ' Tetrahydrofuran Clathrate Hydrate Formation ' , Physical Review Letters , vol. 103 , no. 21 , pp. 218301 . https://doi.org/10.1103/PhysRevLett.

http://hdl.handle.net/10138/27370

https://doi.org/10.1103/PhysRevLett.103.218301

acceptedVersion

Downloaded from Helda, University of Helsinki institutional repository.

This is an electronic reprint of the original article.

This reprint may differ from the original in pagination and typographic detail.

Please cite the original version. 


\title{
Tetrahydrofuran Clathrate Hydrate Formation
}

\author{
Heiko Conrad, ${ }^{1}$ Felix Lehmkühler, ${ }^{1 *}$ Christian Sternemann,,${ }^{1 \dagger}$ Arto Sakko, ${ }^{2}$ Dietmar Paschek, ${ }^{3}$ Laura Simonelli, ${ }^{4}$ \\ Simo Huotari, ${ }^{4}$ Omid Feroughi, ${ }^{1}$ Metin Tolan, ${ }^{1}$ and Keijo Hämäläinen ${ }^{2}$ \\ ${ }^{1}$ Fakultät Physik/DELTA, Technische Universität Dortmund, D-44221 Dortmund, Germany \\ ${ }^{2}$ Department of Physics, P.O. Box 64, FIN-00014 University of Helsinki, Finland \\ ${ }^{3}$ Department of Physics, Applied Physics, and Astronomy, Rensselaer Polytechnic Institute, 110 8th Street, Troy, New York 12180, USA \\ ${ }^{4}$ European Synchrotron Radiation Facility, Boîte Postale 220, F-38043 Grenoble cedex 9, France
}

(Received 27 August 2009; published 19 November 2009)

\begin{abstract}
We report on the formation of tetrahydrofuran clathrate hydrate studied by x-ray Raman scattering measurements at the oxygen $K$ edge. A comparison of x-ray Raman spectra measured from watertetrahydrofuran mixtures and tetrahydrofuran hydrate at different temperatures supports stochastic hydrate formation models rather than models assuming hydrate precursors. This is confirmed by molecular dynamics simulations and density functional theory calculations of x-ray Raman spectra. In addition, changes in the spectra of tetrahydrofuran hydrate with temperatures close to the hydrate's dissociation temperature were observed and may be connected to changes in hydrate's local structure due to the formation of hydrogen bonds between guest and water molecules.
\end{abstract}

DOI: 10.1103/PhysRevLett.103.218301

Clathrate hydrates, i.e., icelike inclusion compounds in which guest molecules are trapped in water nanocages, have reached the focus of general interest owing to a rising number of applications, e.g., methane exhaustion from hydrates or the inhibiting of hydrate formation in pipelines [1]. Tetrahydrofuran (THF) hydrate in particular is a promising candidate for hydrogen storage [2,3]. Pure THF hydrate forms at temperatures below $277.4 \mathrm{~K}$ under atmospheric pressure [4], while the binary hydrate of THF and $\mathrm{H}_{2}$ allows hydrogen storage under industrially attainable conditions, i.e., $p \approx 50$ bar at $T \approx 280 \mathrm{~K}$ [2]. Such applications demand a detailed knowledge of the initial hydrate formation process on molecular length scales. In a recent publication [5], we studied the formation of carbon dioxide hydrate at the water-carbon dioxide interface and concluded that the formation happens in agreement with stochastic models, e.g., the local structuring hypothesis [6]. These models predict that the initial hydrate formation happens if a stochastic arrangement of water and dissolved guest molecules is achieved which is comparable to the hydrate structure. Hydrate precursors, i.e., immediately formed water clusters around guest molecules at conditions allowing hydrate formation, do not exist within the stochastic models. However, the initial hydrate formation process in bulk mixtures such as water-THF has not been studied so far experimentally. In these systems, hydrate precursors may occur in supercooled mixtures of water and hydrate former as predicted by other models such as the cluster nucleation theory [7]. Furthermore, recent molecular dynamics (MD) studies on the polar guests THF and tert-butylmethylether enclosed in water cages showed the formation of hydrogen bonds between guest molecules and water cage [8,9]. These interactions are suggested to lead to broken hydrate structures and may be the reason for an abnormal behavior of thermodynamic
PACS numbers: 82.75.Fq, 61.05.cj, 64.70.D-, 78.70.Ck

properties of THF hydrate close to the hydrate's dissociation temperature [10]. However, such a structural breakup model has also been lacking an experimental verification.

We used x-ray Raman scattering (XRS) to study changes in the local molecular structure of THF hydrate and a 17:1 stoichiometric mixture of water and THF at different temperatures. Changes in the liquid's or hydrate's structure would manifest in modifications in the oxygen $K$ edge fine structure. The temperature dependence of the edges' shape is discussed in the framework of the different formation models for the liquid mixture and with respect to the structural breakup due to strong guest-host interactions for the solid hydrate. The clustering of THF molecules and the formation of hydrate precursors in the liquid phase is studied with MD simulations. Based on molecular structures obtained from these MD simulations, density functional theory (DFT) calculations of XRS spectra are compared with the experimental results.

XRS is a well-established method to study the local structure of both ordered and disordered systems [11]. In XRS measurements, soft $x$-ray absorption edges are examined bulk sensitively using high-energy $x$ rays [12]. XRS has become an important tool to study the coordination of liquids and solids, e.g., water [13,14], or the ordering of water and different ices (see, e.g., Refs. $[15,16])$. This method is highly sensitive to the formation of hydrate precursors in a supercooled mixture of THF and water which would significantly modify the near-edge structure of the oxygen $K$ edge. As the precursors should form everywhere in the sample [7], it affects the coordination and local structure which is accessible by XRS $[11,16]$. A comparison between spectra of the liquid mixture at elevated temperatures and hydrate formation conditions in the supercooled state and of the fully formed hydrate allows us to clarify the hydrate formation process. 
The experiment was performed at beam line ID16 of European Synchrotron Radiation Facility utilizing the multiple-analyzer crystal spectrometer for nonresonant inelastic x-ray scattering spectroscopy [17]. The analyzer energy was fixed to $9.69 \mathrm{keV}$. By tuning the incident energy, energy transfers in the vicinity of the oxygen $K$ edge were achieved. Analyzed x rays were detected employing a Maxipix 2D detector. Using bent $\mathrm{Si}(660)$ analyzer crystals, an overall energy resolution of $0.55 \mathrm{eV}$ was obtained. Spectra for scattering angles $\theta=127^{\circ}-153^{\circ}$ were measured simultaneously yielding momentum transfers of $q=9.01-9.83 \AA^{-1}$. At these high momentum transfers, not only dipole transitions but also nondipole transitions contribute to the spectrum, in contrast to absorption spectroscopy (see, e.g., Ref. [12]). In water systems, for instance, $s-s$ transitions contribute significantly to the XRS spectrum in the vicinity of the preedge whereas $s-p$ transitions dominate mainly in the main and post-edge region [16]. The oxygen $K$ edge region in the energytransfer range of 530-550 eV was measured in approximately $8 \mathrm{~h}$ in each case. In order to estimate the background and the valence electrons' Compton-scattering contribution to the spectrum, the spectra were measured in the energy-loss range of 5-600 eV prior to the oxygen $K$ edge measurement. A specialized sample cell, which allowed an in situ induction of the hydrate growth by stirring the mixture to offer nucleation seeds, was used [18]. A stoichiometric 17:1 mixture of milipore water and THF (Sigma Aldrich, purity $>99.9 \%$ ) was chosen and filled into the cell. To avoid radiation damage [15], the cell was moved to a different position every $10 \mathrm{~s}$ and thus a different part of the sample was exposed by the beam. After the first spectrum from the liquid phase at $T_{1}=293 \mathrm{~K}$ had been finished, the sample was cooled down to the supercooled temperature of $T_{2}=275 \mathrm{~K}$ and the next measurements were performed. Subsequently, the sample was stirred as described in Ref. [18] to offer nucleation seeds for hydrate growth. The temperature was lowered to $T_{3}=274 \mathrm{~K}$ to speed up the hydrate growth. The formation of ice was inhibited by choosing this temperature. The growth of THF hydrate was verified by $x$-ray diffraction indicating the formation of pure THF hydrate. After the hydrate was fully formed, spectra were measured again for several hours at $T_{3}=274 \mathrm{~K}$ and $T_{4}=254 \mathrm{~K}$. The raw spectra were analyzed as described in Ref. [19]. As the $q$ dependence of the XRS spectra is weak for the chosen momentum transfers, spectra for all momentum transfers were summed up to decrease the statistical errors.

The XRS spectra of all measured phases are shown in Fig. 1. The spectra can be subdivided in three regions as indicated in Fig. 1: a preedge region (I) dominated by a maximum at an energy loss of about $535 \mathrm{eV}$, a main edge region (II), and a post-edge region (III) above $539.5 \mathrm{eV}$ energy loss. As the contribution of the sample's local structure to the shape of the preedge is debated controver-

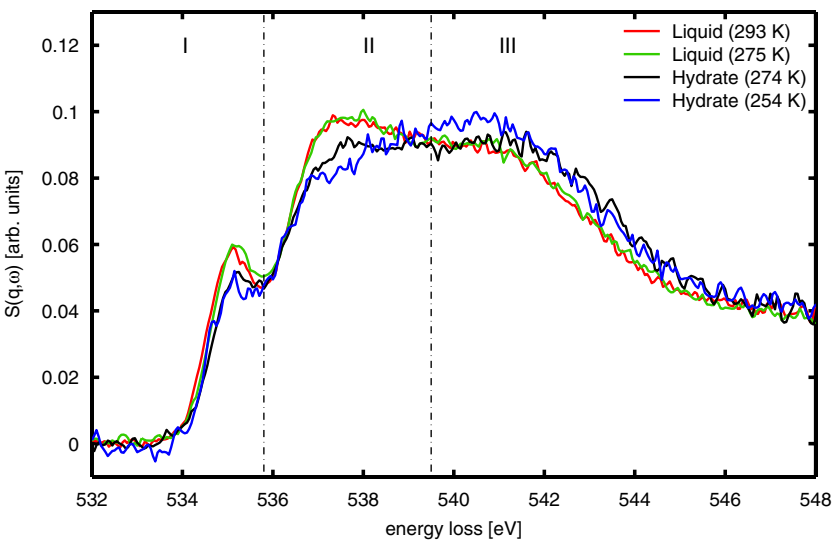

FIG. 1 (color online). Experimental XRS spectra at the oxygen $K$ edge for the liquid mixture and the hydrate at different temperatures. The spectra are divided in a preedge (I), main edge (II), and post-edge (III) region.

sially $[13,15,20]$, our discussion is mainly focused on regions II and III. Here, an increase of structural order, e.g., in the hydrate structure compared to the liquid mixture, or a change of coordination can lead to a shift of spectral weight from the main edge to the post-edge region $[15,16]$. A precursor formation would manifest in such a shift of spectral weight between the liquid mixture at different temperatures. However, the spectra of the liquid mixture at room temperature and supercooled temperature do not show any significant difference (see Fig. 1), in contrast to the spectra of the solid hydrate. Moreover, the increase of spectral weight in region III was observed for the solid hydrate which can be attributed to a previously unreported change in the structure, possibly a rise of structural ordering at lower temperatures. In order to emphasize the changes in the oxygen $K$ edge, differences of the spectra are presented in Fig. 2. For comparison, the averaged difference of water XRS spectra measured at 295 and $275 \mathrm{~K}$ presented in Ref. [14] is also shown to describe the bare temperature effect on bonding in nonsupercooled

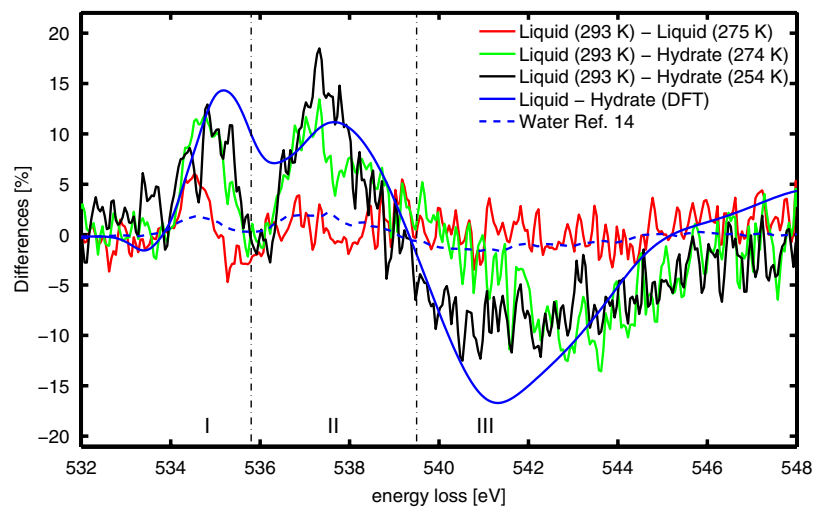

FIG. 2 (color online). Differences of the XRS spectra as indicated. A comparison with calculated spectra (DFT) and the temperature effect of water (Ref. [14]) is also shown. 
water. Thus, the formation of the highly discussed clathratelike structures at supercooled temperatures [21] is suppressed and can be discarded. The deviation in the preedge region (I) can be related to the fact that the water measurements were carried out at a significantly smaller momentum transfer compared to this work, which leads in general to a suppression of the preedge feature and thus to less pronounced differences [16]. Most importantly, in regions II and III the difference between $T_{1}$ and $T_{2}$ of the THF-water mixture is in accordance with the temperature effect of water observed in Ref. [14]. Thus, we conclude that no signatures of hydrate precursors could be found in the supercooled state and that a hydrate formation model such as the local structuring hypothesis is favored by these measurements.

The comparison of the liquid mixture and the hydrate shows a similar general shape as the spectra of water and hexagonal ice $[15,20]$. As mentioned before, the structural ordering of the hydrate compared to the liquid mixture and the similarity of the coordination in hexagonal ice and hydrate [7] is reflected in the disappearance of the maximum in region II and the forming of a new maximum in the post-edge region III. Moreover, the hydrate spectra show a significant difference with temperature that may also be attributed to an increase of structural order of the hydrate at $T_{4}$ compared to $T_{3}$. This finding can be linked to an abnormal behavior of thermodynamic properties of THF hydrate published earlier [10]. It has been observed that the specific heat of THF hydrate shows a nonlinear behavior at temperatures above $T \approx 265 \mathrm{~K}$ which may be connected to a change of hydrate structure, evidenced also by our XRS spectra. The change of the structure above $T \approx$ $265 \mathrm{~K}$ may be due to the formation of hydrogen bonds between THF and water molecules at high temperatures which leads to defects in the hydrate lattice as reported recently [8].

To enable a detailed analysis of the underlying electronic structure behind the spectral features, the experimental results are compared with computational XRS spectra. The structural model for the hydrate phase is obtained from neutron diffraction data presented in Ref. [22]. For the liquid phase, MD simulations were carried out using GROMACS 3.2 [23]. The simulations were performed at a pressure of 1 bar for five temperatures between 250 and $320 \mathrm{~K}$, employing the SPC/E water model [24] and the OPLS-AA force field for THF [25]. However, preliminary simulations indicated a strong tendency of the THF molecules to aggregate and eventually phase separate. This apparently contradicts experimental observations, as the lower critical solution temperature for the THF-water miscibility gap is located at $344 \mathrm{~K}$ [26], well above the temperatures used in our study. Therefore, we decided to modify the intermolecular nonbonded parameters for the THF-THF and THF-water interactions, employing parameters (Lennard-Jones interactions and partial charges) from the TraPPE force field of Siepmann and co-workers [27], which has recently been demonstrated to describe aqueous solutions of polyethers very satisfactorily [28]. These simulations, which were used for analysis, exhibited only a small aggregation tendency of the THF molecules and showed no formation of large THF clusters. Instead, the calculated THF-THF pair correlation functions show similar features as the distribution functions reported from neutron scattering experiments [29]. It should be noted that these simulations do not show evidence for the formation of cluster structures which may act as hydrate precursors. These clusters should form instantly in the supercooled mixture [1] and have lifetimes on the order of the simulated time in our MD simulations [30].

The computational XRS spectra were calculated in the framework of DFT within transition potential approximation utilizing the STOBE-DEMON code that employs localized Gaussian basis functions [31-34]. The method for performing $q$-dependent XRS spectrum calculations [35] within this framework was recently presented in Ref. [31]. The calculated liquid phase spectrum is an average over 135 clusters that were extracted from the MD simulations at $260 \mathrm{~K}$. The MD simulations at the other temperatures yield similar XRS spectra. Each of these clusters was created by randomly selecting an oxygen atom and then including all THF and $\mathrm{H}_{2} \mathrm{O}$ molecules that have at least one atom within a diameter of $d=5.5 \AA$ from the central oxygen. For the hydrate phase calculation a model cluster consisting of several small and large cages was created using diffraction data [22]. THF molecules were fixed into the center of the cages so that their thermal motion was neglected. 18 oxygen-centered clusters with the same radii $(d=5.5 \AA)$ as for the liquid phase were extracted from this structure and their spectra were averaged to obtain the hydrate phase spectrum. Core hole localization was ensured using model core potentials for all nonexcited oxygen sites. To model the lifetime broadening and continuum part of the spectrum, Lorentzian convolution was used with an energy-transfer-dependent half-width of $0.1 \mathrm{eV}$ below $536 \mathrm{eV}$ energy loss and $2.0 \mathrm{eV}$ above $541 \mathrm{eV}$ energy loss. Between 536 and $541 \mathrm{eV}$ energy loss, the half-width increases linearly. Furthermore, a Gaussian convolution with a half-width of the experimental resolution of $0.55 \mathrm{eV}$ was also applied. A gradient corrected exchange correlation functional [36,37] was employed in the calculations. In order to match the experimental data, the spectra had to be shifted by $-1.2 \mathrm{eV}$. Results are shown in Fig. 2 .

A good overall agreement of experiment and theory is achieved, in particular, for the hydrate at $T_{4}=254 \mathrm{~K}$ compared to the difference of liquid and hydrate at $T_{3}=$ $274 \mathrm{~K}$ near the dissociation temperature. The difference between $T_{3}$ and $T_{4}$ indicates that the formation of guesthost bonds is possibly suppressed at lower temperatures as predicted by Alavi et al. [8]. The lack of hydrate precursors in the MD simulations and the good agreement of experi- 
ment and DFT calculations strongly supports the absence of hydrate precursors in a supercooled mixture.

In conclusion, the XRS measurements at the oxygen $K$ edge of water-THF mixtures and THF hydrate reveals detailed information of the microscopic hydrate formation process in a bulk mixture of water and THF. The use of XRS together with MD simulations and DFT calculations gives a deeper understanding of the local molecular structure of a hydrate former-water mixture. The results support stochastic hydrate formation rather than precursor based theories. Furthermore, the abnormal behavior of thermodynamic properties of THF hydrate can be interpreted in a change of long range ordering with temperature originated in a strong interaction between water and THF. Both findings may have a great impact for the research of hydrates in general and for applications for gas storage, e.g., hydrogen, in particular.

The authors thank M. Hakala, J.A. Soininen, and T. Pylkkänen for discussions and the ESRF for providing synchrotron radiation. This work was funded in part by the DFG (TO 169/12-1), the BMBF (05 KSPE1 and 05 KSPEA), the DAAD (313-PPP-SF-08-Ik and 1127504), and the Academy of Finland (1110571 and 1127462).

*Felix.Lehmkuehler@tu-dortmund.de

Christian.Sternemann@tu-dortmund.de

[1] E. D. Sloan, Nature (London) 426, 353 (2003).

[2] L. J. Florusse, C. J. Peters, J. Schoonman, K. C. Hester, C. A. Koh, S.F. Dec, K. N. Marsh, and E.D. Sloan, Science 306, 469 (2004).

[3] H. Lee, J. Lee, D. Y. Kim, J. Park, Y. Seo, H. Zeng, I. L. Moudrakovski, C.I. Ratcliffe, and J.A. Ripmeester, Nature (London) 434, 743 (2005).

[4] T. Makino, T. Sugahara, and K. Ohgaki, J. Chem. Eng. Data 50, 2058 (2005).

[5] F. Lehmkühler, M. Paulus, C. Sternemann, D. Lietz, F. Venturini, C. Gutt, and M. Tolan, J. Am. Chem. Soc. 131, 585 (2009).

[6] R. Radhakrishnan and B. L. Trout, J. Chem. Phys. 117, 1786 (2002).

[7] E. D. Sloan and C. A. Koh, Clathrate Hydrates of Natural Gases (CRC Press, Boca Raton, 2007).

[8] S. Alavi, R. Susilo, and J. A. Ripmeester, J. Chem. Phys. 130, 174501 (2009).

[9] R. Susilo, S. Alavi, I. L. Moudrakovski, P. Englezos, and J. A. Ripmeester, Chem. Phys. Chem. 10, 824 (2009).

[10] W. F. Waite, L. Y. Gilbert, W. J. Winters, and D. H. Mason, Proceedings of the Fifth International Conference on Gas Hydrates (Tapir Academic Press, Trondheim, 2005), p. 1725.

[11] W. Schülke, Electron Dynamics by Inelastic X-Ray Scattering (Oxford University Press, Oxford, 2008).

[12] C. Sternemann, H. Sternemann, S. Huotari, F. Lehmkühler, M. Tolan, and J.S. Tse, J. Anal. At. Spectrom. 23, 807 (2008) and references therein.
[13] Ph. Wernet, D. Nordlund, U. Bergmann, H. Ogasawara, M Cavalleri, L. A. Näslund, T. Hirsch, L. Ojamäe, P. Glatzel, M. Odelius, L. G. M. Pettersson, and A. Nilsson, Science 304, 995 (2004).

[14] U. Bergmann, D. Nordlund, Ph. Wernet, M. Odelius, L. G. M. Pettersson, and A. Nilsson, Phys. Rev. B 76, 024202 (2007).

[15] J.S. Tse, D. M. Shaw, D. D. Klug, S. Patchkovskii, G. Vankó, G. Monaco, and M. Krisch, Phys. Rev. Lett. 100, 095502 (2008).

[16] T. Pylkkänen, V. M. Giordano, J.-C. Chervin, A. Sakko, M. Hakala, J. A. Soininen, K. Hämäläinen, G. Monaco, and S. Huotari (to be published).

[17] R. Verbeni, T. Pylkkänen, S. Huotari, L. Simonelli, G. Vankó, K. Martel, C. Henriquet, and G. Monaco, J. Synchrotron Radiat. 16, 469 (2009).

[18] H. Conrad, F. Lehmkühler, C. Sternemann, O. Feroughi, L. Simonelli, S. Huotari, and M. Tolan, Rev. Sci. Instrum. 80, 026103 (2009).

[19] H. Sternemann, C. Sternemann, G. T. Seidler, T. T. Fister, A. Sakko, and M. Tolan, J. Synchrotron Radiat. 15, 162 (2008).

[20] Y. Q. Cai et al., Phys. Rev. Lett. 94, 025502 (2005).

[21] H. Yokoyama, M. Kannami, and H. Kanno, Chem. Phys. Lett. 463, 99 (2008).

[22] C. Y. Jones, S. L. Marshall, B. C. Chakoumakos, C. J. Rawn, and Y. Ishii, J. Phys. Chem. B 107, 6026 (2003).

[23] E. Lindahl, B. Hess, and D. van der Spoel, J. Mol. Model. 7, 306 (2001).

[24] H. J.C. Berendsen, J. R. Grigera, and T. P. Straatsma, J. Phys. Chem. 91, 6269 (1987).

[25] W. L. Jorgensen, D. S. Maxwell, and J. Tirado-Rives, J. Am. Chem. Soc. 118, 11225 (1996).

[26] A. Oleinikova and H. Weingärtner, Chem. Phys. Lett. 319, 119 (2000).

[27] J. M. Stubbs, J. J. Potoff, and J. I. Siepmann, J. Phys. Chem. B 108, 17596 (2004).

[28] J. Fischer, D. Paschek, A. Geiger, and G. Sadowski, J. Phys. Chem. B 112, 2388 (2008).

[29] D. T. Bowron, J. L. Finney, and A. K. Soper, J. Phys. Chem. B 110, 20235 (2006).

[30] G.-J. Guo, Y.-G. Zhang, Y.-J. Zhao, K. Refson, and G.-H. Shan, J. Chem. Phys. 121, 1542 (2004).

[31] A. Sakko, M. Hakala, J. A. Soininen, and K. Hämäläinen, Phys. Rev. B 76, 205115 (2007).

[32] L. Triguero, L. G. M. Pettersson, and H. Ågren, Phys. Rev. B 58, 8097 (1998).

[33] K. Hermann et al., computer code STOBE-DEMON version 3.0, available at http://w3.rz-berlin.mpg.de/ hermann/ StoBe/index.html.

[34] In this work, the iii-iglo basis set, together with a set of diffuse basis functions, was employed for the excited oxygen site and the triple zeta plus valence polarization level basis sets for the other atoms.

[35] J. A. Soininen, A. L. Ankudinov, and J. J. Rehr, Phys. Rev. B 72, 045136 (2005).

[36] B. Hammer, L. B. Hansen, and J. K. Nørskov, Phys. Rev. B 59, 7413 (1999).

[37] J. P. Perdew, K. Burke, and M. Ernzerhof, Phys. Rev. Lett. 77, 3865 (1996). 